\title{
The Path from Basic Research to Clinical Practice
}

\author{
Chunyu Liu ${ }^{1 *}$, Elliot S. Gershon ${ }^{2}$ \\ ${ }^{1}$ Department of Psychiatry, University of Illinois at Chicago. \\ ${ }^{2}$ Department of Psychiatry and Behavioral Science, The University of Chicago.
}

*Corresponding to: Chunyu Liu. liucy@uic.edu .

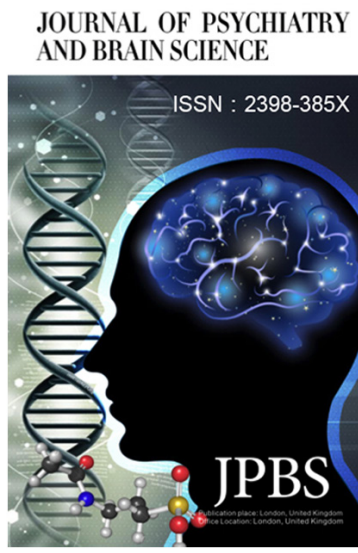

http://jpbs.qingres.com

\section{fOPEN ACCESS}

DOI: 10.20900/jpbs.20170007

Received: January 28, 2017

Accepted: March 26, 2017

Published: April 25, 2017

Copyright: $\odot 2017$ Cain et al. This is an open access article distributed under the terms of the Creative Commons Attribution License, which permits unrestricted use, distribution, and reproduction in any medium, provided the original author and source are credited.
Dr. Belmaker presents his opinion on an important issue, how much a basic research result can be used to guide clinical practice ${ }^{[1]}$. Dangerous traps in the process from basic research to bed-side practice exist, particularly in the time of "new media." When patients are in desperate struggles looking for better treatment of their disorders, their decisions may be misled by news, become less logical, and ultimately make their situation worse. This is a particular problem for alternative medicine. Clinicians can provide important safeguards against these worsened outcomes.

Ionnidis has reviewed the problem of non-replicability of many scientific findings, pointing to the needs of cautious use of research results ${ }^{[2]}$. Well-designed clinical trials, with large sample sizes, and replicated findings are the gold standard for results that can be applied to patients.

The media also need to take responsibility for the scientific significance of the basic research results they publicize, so that they are not exaggerated beyond what they can truly represent. Even though the ultimate choice of a treatment lies in the hands of our patients, clinicians bear the responsibility to provide education and informed advice about their choices.

\section{REFERENCES}

1. Belmaker RH. When Do Good Research Ideas Become Clinically Relevant. Journal of Psychiatry and Brain Science. 2017; 2(2): 1; DOI: 10.20900 / jpbs. 20170003.

2. Ioannidis JP. Why most published research findings are false. PLoS Med. 2007; 4(6): e215. 